\title{
Perfil Antropométrico y Aptitud Física de Árbitros del Fútbol Profesional Chileno
}

\author{
Anthropometric Profile and Physical Fitness of the Professional Referees Chilean Soccer \\ Germán Esteban Fernández Vargas; Alberto Inácio da Silva \& Miguel Arruda
}

FERNÁNDEZ, V. G. E.; DA SILVA, A. I. \& ARRUDA MIGUEL. Perfil antropométrico y aptitud física de árbitros del fútbol profesional chileno. Int. J. Morphol., 26(4):897-904, 2008.

RESUMEN:El objetivo del presente estudio fue analizar el estado el perfil antropométrico y la aptitud física en árbitros de primera categoría, participantes del fútbol profesional chileno, durante la temporada 2007. La muestra fue constituida por 11 árbitros principales, todos de sexo masculino. El promedio de edad fue de 34,54 \pm 4,76 años, estatura 1,74 \pm 0,05 m, masa corporal 76,95 $\pm 5,75$ $\mathrm{kg}$. Las variables de masa corporal, estatura, grosor de pliegues cutáneos, circunferencias corporales y diámetros óseos, fueron medidos con la finalidad de estimar la composición corporal y determinar el somatotipo de los árbitros. Para determinar la composición corporal se utilizaron: 7 pliegues cutáneos, 7 circunferencias corporales y 4 diámetros óseos. Para evaluar las variables relacionadas con la aptitud física se utilizó la nueva batería de tests físicos de la FIFA, que determina evaluar al árbitro en primera instancia en cuanto a su capacidad anaeróbica al ejecutar carreras rápidas $(6 \mathrm{x} 40 \mathrm{~m})$ y a su vez medir su capacidad de potencia aérobica en la segunda prueba. Con respecto a los datos de composición corporal, los resultados indicaron que el porcentaje de grasa fue de $15,44 \pm 2,81 \%$, con un somatotipo promedio de 3,81 - 5,67 - 1,57, clasificados en meso-endomorfo y un 25,14 $\pm 1,18 \mathrm{~kg} / \mathrm{m}^{2}$ de IMC. Los resultados relacionados a la aptitud física mostraron que los árbitros chilenos cubrieron, en promedio, 5,32 \pm 0,02 segundos el test anaeróbico, mientras que en el segundo, todos cubrieron cómodamente la distancia de $4.000 \mathrm{~m}$. La mayor acumulación de grasa en árbitros profesionales puede ser una limitante del rendimiento físico durante el partido, lo cual puede ser controlada a través de programas de alimentos y entrenamiento físico.

PALABRAS CLAVE: Árbitro; Fútbol; Antropometría.

\section{INTRODUCCIÓN}

Cobra vital relevancia en la práctica deportiva del fútbol, no tan sólo la actuación de los involucrados directos en el espectáculo, como lo son los jugadores, sino que además el equipo de árbitros.

Aquellos considerados responsables directos en la implementación de las reglas de juego mediante la aplicación de éstas, que de acuerdo a las normativas establecidas por el organismo rector del fútbol a nivel mundial, las cuales en acciones propias del juego, sus fallos son calificados definitivos (FIFA, 2007).

Es naturalmente indudable que a la luz de cualquier tipo de espectáculo futbolístico, éste es un deporte físicamente muy agotador. Cualquier profesional de las ciencias de la actividad física y deportiva puede reconocer esta idea. Trabajos de origen científico, involucrando árbitros de fút- bol son muy recientes y escasos, si tomamos como referencia los estudios involucrando jugadores de fútbol (Asami et al., 1988; Catterall et al., 1993; Da Silva \& Rodríguez-Añez, 1999; D’Ottavio \& Castagna, 2001).

Los estudios desarrollados con árbitros son muy variables. Buena parte de los trabajos describen las acciones motoras del árbitro de fútbol durante un partido, con las respectivas distancias recorridas (Johnston \& McNaughton, 1994; Da Silva \& Rodríguez-Añez, 1999; Castagna et al., 2004). Otros trabajos abordan los parámetros antropométricos (Rontoyannis et al., 1998; Da Silva \& Rodríguez-Añez, 2003). La intensidad de la actividad física del árbitro durante el partido fue estudiada por Roman et al., (2004); Da Silva \& Rodriguez-Añez, (2007). También ha sido hecha la cuantificación de la deshidratación del árbitro y del árbitro asistente durante partidos oficiales (Da Silva \&

Programa de Posgrado de la Universidad de Tarapacá, Chile

Prof. Asistente de la Universidad Estadual de Ponta Grossa, UEPG - Paraná, Brasil

Prof. Titular de la Universidad Estadual de Campinas, Unicamp - São Paulo, Brasil 
Fernández, 2003) y más recientemente fue determinado el gasto calórico del árbitro en el transcurso del partido (Da Silva et al., 2008). A partir del año 2000, por lo tanto muy recientemente, empezaron a surgir estudios académicos dirigidos a la preparación física de los árbitros (Krustrup \& Bangsbo, 2001; Da Silva et al., 2002; Rebelo et al., 2002; Weston et al., 2004).

Así como hubo una gran evolución en el entrenamiento deportivo, ocurrió también una gran evolución en las formas de medir los resultados obtenidos durante un programa de entrenamiento. Tests físicos que expresan el nivel de las capacidades físicas utilizadas por los árbitros en el transcurrir de un partido de fútbol, están siendo objeto de estudio y sugeridos por la bibliografía científica (Krustrup \& Bangsbo, 2001; Castagna et al., 2002; Castagna et al., 2005; Da Silva, 2005). El año 2006, la Fédération International de Football Association (FIFA) estableció nuevas pruebas anaeróbicas, y aeróbicas para sus colegiados, contempladas en su renovada batería de tests físicos.

Para cumplir una buena función, no tan sólo necesitan un buen desempeño técnico, sino que también una buena performance física y una apariencia atlética. De acuerdo con Cuchiaro (2000), con el fin de establecer programas específicos de entrenamiento, es necesario conocer el perfil antropométrico del atleta para saber si éste está sobre o bajo el perfil adecuado para la función, además de las exigencias físicas de la actividad. Se sabe aún que cantidades elevadas de grasa corporal perjudican el desempeño de los individuos, junto con constituir un factor de riesgo para diversas enfermedades. Por lo tanto, es fundamental el control adecuado de la adiposidad corporal.

Además de las medidas de composición corporal (grasa corporal, masa corporal magra), un excelente indicador de la forma, estructura y composición del cuerpo humano se refiere a la técnica del somatotipo. Esa técnica constituye un recurso extremadamente útil para el análisis de las modificaciones en la forma y estructura corporales en función del entrenamiento, o por la propia exigencia física de la actividad en cuestión (Carter et al., 2005).

De los pocos trabajos que abordan el resultado físico de los árbitros de fútbol, en los tests determinados por la FIFA no encontramos ningún relato científico sobre la capacidad física frente a la nueva secuencia de la aplicación de los tests que integran la batería de ellos de esta entidad. Esta ausencia de artículos puede ser explicada, tal vez por la reciente modificación (año 2006) en la aplicación de los tests y por la restricción a investigadores a tener acceso a datos que involucran la evaluación física de árbitros profesionales.
Sin embargo, en los trabajos que abordan las exigencias físicas del árbitro en el transcurso del partido de fútbol y el perfil antropométrico, es prácticamente escasa o nula la literatura encontrada sobre la clasificación física (somatotipo) de este profesional. Infiriendo que las demandas energéticas de los árbitros son próximas o cercanas a las presentadas por los jugadores de fútbol (Da Silva et al., 2008), por cuanto es de esperar que su tipo físico sea semejante.

Se considera necesario realizar estudios que aborden el perfil antropométrico (somatotipo) y el nivel de capacidad física de los árbitros de fútbol. Así se evitaría que decisiones equivocadas provocadas por agotamiento físico durante el partido resten brillo de un buen juego de fútbol. Por consiguiente, el objetivo de este estudio es analizar el perfil antropométrico, determinar el somatotipo y la aptitud física en árbitros de primera categoría del fútbol profesional chileno.

\section{METODOLOGÍA}

Los procedimientos adoptados en este estudio están de acuerdo con las técnicas adecuadas descritas en la literatura y no implican riesgos o perjuicios a los evaluados.

Antes del inicio de la recolección de los datos, fueron informados los propósitos y procedimientos técnicos que serian adoptados, enfocando los objetivos y beneficios de la investigación para los participantes, a través de una reunión del responsable de la investigación, junto con el preparador físico de los árbitros, así como de toda la muestra de estudio. En esta reunión fue garantizada la libertad de asistencia de participación en el estudio por parte de los árbitros antes y durante las evaluaciones.

La muestra de estudio fue compuesta por 11 árbitros principales, participantes en el campeonato "Copa Banco Estado" año 2007, de la primera división "A" del fútbol profesional chileno, donde se destacan cuatro árbitros de categoría internacional, considerada una distinción entregada por FIFA. Los sujetos a evaluar eran todos de sexo masculino, de edad media de 34,54 $\pm 4,76$ años, con una estatura media de 1,74 \pm $0,05 \mathrm{~m}$, un peso promedio de $76 \pm 5,75 \mathrm{Kg}$ y pertenecientes a la comisión de árbitros profesionales de Chile.

Para la evaluación de la composición corporal fueron medidos: 7 pliegues cutáneos (tríceps, bíceps, subescapular, abdominal, suprailiaco, muslo y pantorrilla)utilizando un adipómetro marca Harpenden ${ }^{\circledR}$ de fabricación Británica, modelo HSK-BI, con una precisión de medida de $0,2 \mathrm{~mm}$ y una presión constante sobre la piel 
de $10 \mathrm{~g} / \mathrm{mm}^{2}$. También se midieron 7 circunferencias corporales (antebrazo, brazo, brazo contraído, abdominal, cadera, muslo y pantorrilla) utilizando una cinta métrica marca Lufkin ${ }^{\circledR}$, modelo W606PM, con una graduación de $0,1 \mathrm{~cm}$. A su vez, se realizó la medición de 4 diámetros óseos (biestiloide, biepicondiliano del húmero, biepicondiliano del fémur y bimaleolar), empleando un antropómetro o calibre óseo, marca Mitutoyo®, modelo CD-S6"C con una precisión de $0,1 \mathrm{~cm}$.

A la evaluación de estas variables antropométricas (pliegues cutáneos, circunferencias corporales y diámetros óseos), siguieron las estandarizaciones propuestas por Harrison et al. (1991), Callaway et al. (1991) y Wilmore et al. (1991).

Para el presente estudio el porcentaje de grasa $(\% \mathrm{G})$ fue determinado a través de la ecuación propuesta por Yuhasz (1962) y luego modificada por Faulkner (1968).

Para fines de comparación, los somatotipos fue clasificado en categorías de acuerdo con Carter (2002). El somatotipo también fue expuesto en un gráfico (somatocarta), desarrollada por Carter \& Heath (1990), donde fueron calculados los valores de las coordenadas $\mathrm{X}$ y $\mathrm{Y}$ : $\mathrm{X}=$ ectomorfia - endomorfia; $\mathrm{Y}=2 \times$ mesomorfia - (endomorfia + ectomorfia).

Los tests físicos fueron aplicados siguiendo el protocolo establecido por las dos pruebas determinadas por el organismo rector del fútbol a nivel mundial (FIFA). La prueba número uno tenía como objetivo medir la velocidad de desplazamiento. El árbitro debe realizar 6 carreras de $40 \mathrm{~m}$, con un minuto de recuperación después de cada una. La prueba número dos tenía como objetivo medir la capacidad de rendimiento de la potencia aeróbica en repetidas carreras. Los árbitros deben cubrir una distancia de $150 \mathrm{~m}$ de carrera en 30 segundos y luego disponen de 35 segundos para cubrir una distancia de $50 \mathrm{~m}$ de caminata, hasta cubrir la distancia de 10 vueltas de $400 \mathrm{~m}$.

Una vez registrados los datos de la aplicación del primer test, se utilizó la fórmula empleada por el test Running-Based Anaerobic Sprint Test (R.A.S.T.), para así determinar: velocidad, aceleración, fuerza y potencia, durante la realización de la carrera de 40 metros (Draper \& Whyte, 1997).

Para el tratamiento estadístico de los datos, se utilizó inicialmente la estadística descriptiva para agrupar los resultados en valores promedio con su desviación. El coeficiente de correlación de Pearson fue calculado para determinar el grado de correlación entre variables específicas.

\section{RESULTADOS}

En la Tabla I son presentadas las características antropométricas de los árbitros chilenos. Apenas el $46 \%$ de los ellos están con el IMC dentro del valor de normalidad $\left(25 \mathrm{~kg} / \mathrm{m}^{2}\right)$, que fue de $24,2 \pm 0,6 \mathrm{~kg} / \mathrm{m}^{2}$. El restante $54 \%$ de los árbitros, presentan un IMC por encima de la normalidad que fue de $26 \pm 0,9 \mathrm{~kg} / \mathrm{m}^{2}$.

En cuanto a las características somatotipológicas de los árbitros principales, los resultados determinaron que 9 sujetos $(81,81 \%$ de la muestra) clasificaron como mesoendomorfos, mientras que un sujeto $(9,09 \%)$ era mesoectomorfo y un sujeto $(9,09 \%)$ era endo-mesomorfo.

La media indicó que los árbitros evaluados fueron clasificados mediante este método como meso-endomorfos, ya que registraron un valor medio de 3,81 en el componente endomorfo, 5,67 en el componente mesomorfo y un 1,57 en el componente ectomorfo.

Tabla 1. Características antropométricas de los sujetos de estudio

\begin{tabular}{lcccc}
\hline & Media & DS & Mayor valor & Menor valor \\
\hline Edad (años) & 34,54 & 4,76 & 43 & 28 \\
Estatura & 1,74 & 0,05 & 1,82 & 1,67 \\
Masa corporal $(\mathrm{kg})$ & 76,95 & 5,75 & 88,7 & 71,2 \\
IMC $\left(\mathrm{kg} / \mathrm{m}^{2}\right)$ & 25,14 & 1,18 & 26,78 & 23,50 \\
Grasa corporal $(\%)$ & 15,44 & 2,81 & 19,24 & 10,34 \\
Peso grasa $(\mathrm{kg})$ & 11,92 & 2,57 & 16,14 & 7,93 \\
Peso óseo $(\mathrm{kg})$ & 15,32 & 0,69 & 16,30 & 14,38 \\
Peso residual $(\mathrm{kg})$ & 18,54 & 1,38 & 21,37 & 17,15 \\
Peso muscular $(\mathrm{kg})$ & 31,16 & 2,91 & 36,33 & 27,64 \\
\hline
\end{tabular}


La Fig. 1 exhibe una somatocarta con la distribución de somatopuntos registrados por los árbitros chilenos.

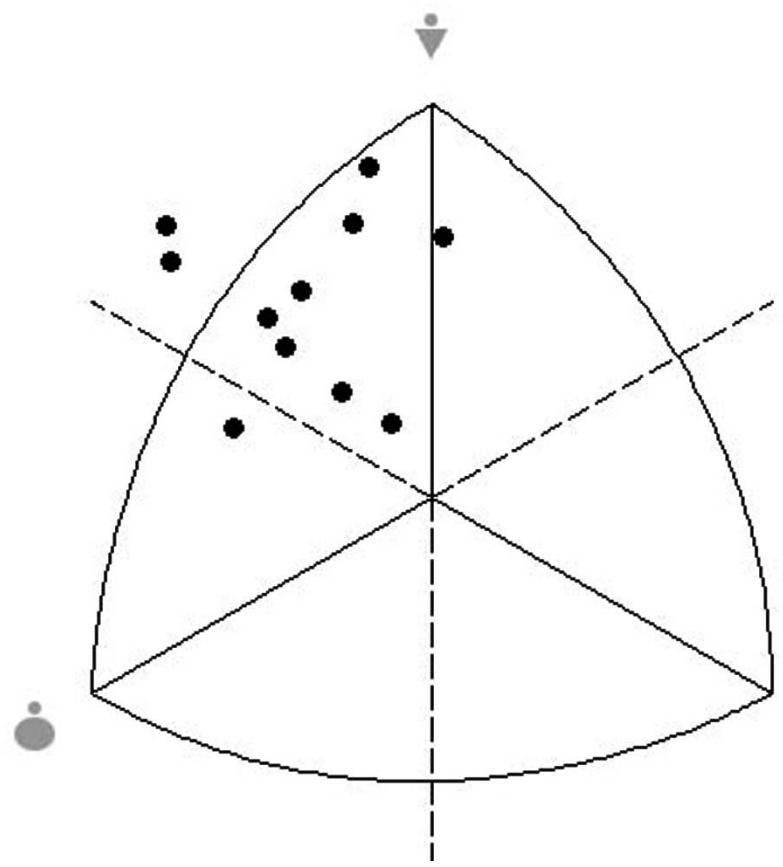

Fig. 1. Somatocarta con distribución de somatopuntos de los árbitros del fútbol profesional chilenos.

El valor promedio presentado en la Tabla II fue obtenido por los árbitros durante la ejecución del test anaeróbico correspondiente a 6 carreras de $40 \mathrm{~m}$. Se pudo observar que los árbitros chilenos, en comparación con los tiempos interpuestos por FIFA, estaban dentro del límite del tiempo exigido $(6,4 \mathrm{seg})$. Destaca aún, que todos los árbitros del presente estudio, en cada una de las carreras, ninguno registró un tiempo por encima del límite impuesto para la prueba.

Tabla II. Registros de tiempos (seg) obtenidos en la prueba anaeróbica de la FIFA.

\begin{tabular}{lcccc} 
& Media & DS & $\begin{array}{c}\text { Mayor } \\
\text { valor }\end{array}$ & $\begin{array}{c}\text { Menor } \\
\text { valor }\end{array}$ \\
$\begin{array}{lccc}\text { Prueba } \\
(6 \times 40 \mathrm{~m})\end{array}$ & 5,32 & 0,01 & 5,72 & 4,97 \\
\hline
\end{tabular}

Por intermedio de la fórmula de R.A.S.T. test es posible conseguir los valores en relación a: velocidad, aceleración, fuerza y potencia de los sujetos en sus grados máximo, medio y mínimo. Los valores promedios de cada una de estas variables se presentan en la Tabla III.

La última prueba recomendada por la FIFA para evaluar la aptitud física de sus colegiados, considera la ejecu- ción de 10 vueltas a la pista de atletismo considerando para la categoría de la muestra (árbitros nacionales) un tiempo de $30 \mathrm{seg}$, corriendo una distancia de $150 \mathrm{~m}$, para luego recuperarse en $50 \mathrm{~m}$ en $35 \mathrm{seg}$. Cabe destacar que para la presente evaluación física todos los sujetos investigados ejecutaron sin mayores problemas el test, registrando la cantidad deseada de metros recorridos de 4.000.

\section{DISCUSIÓN}

El valor promedio de la masa corporal de los árbitros de Chile fue de 76,9 $\pm 5,7 \mathrm{~kg}$. Esta cifra resultó similar a la media de los árbitros griegos (Rontoyannis et al.) y de los árbitros brasileños (Roman; Da Silva \& Nacimento, 2005).

Con respecto a la estatura, la altura media registrada (Tabla I) también mostró valores similares a los de la literatura especializada (Rontoyannis et al.; Da Silva \& Nacimento).

El promedio de edad encontrado en este estudio fue de 34,54 \pm 6,76 años, apreciándose este valor en concordancia con algunos estudios publicados con árbitros de fútbol de otras latitudes (Rontoyannis et al.; Betsch \& Plessner, 2001; Rebelo et al., 2002; Da Silva \& Fernández, 2003). Algunas investigaciones concluyen que los árbitros de fútbol son, en promedio, de 10 a 15 años mayores que los jugadores de élite de este deporte y que la edad tendría efectos negativos en el rendimiento físico. Los árbitros que participaron en este estudio no escaparon de esa norma (Catterall et al., 1993; Weston et al., 2004; Helsen \& Bultynck, 2004).

Cuando se asocia el peso y la altura al cuadrado se establece un nuevo índice conocido como el IMC. Con el agrupamiento de los dados y aplicación de la formula para determinación del IMC, se verificó que el valor promedio del IMC de los árbitros chilenos fue de $25,1 \pm 1,8 \mathrm{~kg} / \mathrm{m}^{2}$, o sea, un poco por encima del valor de normalidad. El IMC de 220 árbitros brasileños, no fue diferente de la media de (24,87 $\pm 2,86 \mathrm{~kg} / \mathrm{m}^{2}$, Da Silva, 2006). Sin embargo, la concentración de grasa de estos árbitros era de 18,56 $\pm 4,29 \%$. En otro estudio, involucrando ahora sólo árbitros de elite de Brasil, se observó que el IMC promedio fue de 25,38 $\pm 2,27 \mathrm{~kg} / \mathrm{m}^{2}$, donde el \%G era de 19,9\% (Da Silva et al., 2007).

El grupo de árbitros chilenos presentó un $\% \mathrm{G}$ de un $15,4 \pm 2,8 \%$, muy semejante al de sus similares de elite de España de un $11,3 \pm 2,1 \%$, y en menor porcentaje al encontrado por nosotros en la literatura científica (Casajus \& Castagna, 2006). En una investigación conducida con 
FERNÁNDEZ, V. G. E.; DA SILVA, A. I. \& ARRUDA MIGUEL. Perfil antropométrico y aptitud física de árbitros del fútbol profesional chileno. Int. J. Morphol., 26(4):897-904, 2008.

Tabla III. Registro descriptivo de la prueba física 1 al aplicar la formula de R.A.S.T.

\begin{tabular}{lcccc}
\hline & MEDIA & DS \pm & Mayor valor & Menor valor \\
Velocidad $(\mathrm{m} / \mathrm{s})$ & 7,52 & 0,02 & 8,04 & 6,98 \\
Aceleración $\left(\mathrm{m} / \mathrm{s}^{2}\right)$ & 1,41 & 0,01 & 1,61 & 1,22 \\
Fuerza $\left(\mathrm{m} / \mathrm{s}^{2} \_\mathrm{kg}\right)$ & 109,23 & 1,40 & 139,59 & 90,48 \\
Potencia (watt) & 825 & 14,94 & 876 & 776 \\
Índice de fatiga $(\mathrm{w} / \mathrm{s})$ & 3,16 & 1,39 & 5,73 & 1,35 \\
\hline
\end{tabular}

atletas del fútbol brasileño, realizado por Guerra et al., (2004) se observó por medio del método antropométrico, un valor de adiposidad de $10,6 \%$, encontrando valores iguales $(10,6 \pm 2,6 \%)$ en jugadores de países de América del Sur (Rienzi et al., 1998). Esos valores son inferiores a los presentados por gran parte de los árbitros profesionales. Eso demuestra que, con relación al acúmulo de adiposidad, los árbitros evaluados están en condiciones físicas inferiores al compararlos con atletas de esta modalidad.

Con relación al somatotipo, la media indicaba que los árbitros evaluados fueron clasificados mesoendomorfos, ya que registraron un valor medio de 3,8-5,61,5 (Fig. 1) aunque los árbitros presentaron $15,44 \%$, de grasa.

Rahmawati et al. (2006), analizaron un grupo de atletas de diferentes modalidades, entre ellos de fútbol y encontraron un somatotipo medio meso-endomorfo $(2,7$ 4,9-2,9), vale decir una clara característica de predominio del desarrollo muscular sobre el componente adiposo y lineal (estatura). En el grupo de árbitros parece lógico que la estatura no tenga una influencia directa en la actuación de los mismos, sin embargo, el componente muscular y adiposo están directamente relacionados a la performance física durante del juego.

A continuación se presentan los resultados arrojados por el análisis estadístico descriptivo al ser aplicada la correlación de Pearson a las diferentes variables de estudio. Cabe señalar que para la presente investigación serán obviados aquellas correlaciones que aún presentando una fuerte correlación no tengan incidencia directa entre variables que guarden una misma relación.

Después del análisis entre las características antropométricas de los sujetos con las características de la aptitud física (prueba 1), fue posible observar que el peso muscular promedio de los árbitros chilenos se relacionaba considerablemente con la fuerza $(r=0,82)$, con la potencia máxima $(r=0,78)$ y con la potencia media $(r=0,76)$ ejercida en la prueba 1 .
Cuando el coeficiente $r$ de Pearson se eleva al cuadrado (r2), el resultado indica la varianza de factores comunes. Esto es, el porcentaje de la variación de una variable debido a la variación de la otra variable y viceversa. De esta manera se puede explicar o interpretar que el peso muscular constituye o explica el $67 \%$ de la fuerza ejercida en la prueba física 1, así también representa o explica el $61 \%$ de la potencia máxima y por último el peso muscular representa o explica el $58 \%$ de la potencia media. Estudios recientes destacan la importancia del desarrollo de la fuerza muscular y la potencia durante el entrenamiento, para que el árbitro presente desplazamientos con mayor velocidad durante el partido (Krustrup \& Bangsbo, 2001; Weston et al.).

La segunda prueba de los tests de la FIFA, como se describió anteriormente, está constituida por 20 carreras de 150 metros, intercalada por 20 caminatas de 50 metros. Los tiempos para cada carrera o caminata son invariables. De esta manera no se puede verificar específicamente si algún árbitro está mejor que otro físicamente. Otro problema que observamos, es que el resultado de este test no permite determinar ninguna variable fisiológica. Como a su vez, se observa que las exigencias físicas impuestas a este nivel de árbitros nacionales, no ofrecen un grado de dificultad mayor de acuerdo a los protocolos impuestos por FIFA. Anterior a este test, era aplicado el test de Cooper (corrida de 12 minutos) que, dependiendo de la distancia recorrida se podría estimar el $\mathrm{VO}_{2}$ máx. del árbitro o clasificar su aptitud física según el protocolo de Cooper. Aún los resultados presentados por los árbitros durante la carrera de 12 minutos presentaba correlación de $\mathrm{r}=0,71$ con la distancia recorrida por el árbitro durante el partido (Castagna et al., 2002). Se considera de esta forma que el test actual elaborado no permite este tipo de análisis.

Otra propuesta encontrada en la literatura científica para la evaluación de la capacidad aeróbica del árbitro durante el juego, fue hecha por Krustrup \& Bangsbo. Estos autores encontraron una fuerte correlación entre el Yo-Yo test de recuperación intermitente (descrito por Krustrup et al., 2003) y las acciones motoras de alta velocidad $(\mathrm{r}=0,75)$ y una correlación significativa entre la distancia total $(r=0,66)$ ejecutada por los árbitros durante el partido. Da Silva (2005) 
sugiere la utilización del test de multi-estadio de 20 metros de Léger et al., pues el $\mathrm{VO}_{2}$ máx obtenido durante este test versus el test de Cooper no presentaban diferencias estadísticamente significativas. Consideraciones compartidas por nosotros puesto que los resultados son individuales, ya que permiten obtener una variable fisiológica y distinguir los árbitros de acuerdo con su capacidad física, datos necesarios para el desarrollo de programas de entrenamiento físico y nutricional específicos para los árbitro de fútbol.
En las fuentes investigadas no fueron encontradas discusiones profundas sobre la necesidad de medir la capacidad anaeróbica del árbitro. Krustrup \& Bangsbo; Weston et al. y Da Silva, (2005) sugieren que los árbitros sean sometidos a un programa de entrenamiento específico, con sesiones de entrenamiento con cargas intermitentes de alta intensidad, siendo esa prescripción suficiente para desarrollar las fuentes energéticas utilizadas por los árbitros de fútbol durante el juego.

FERNÁNDEZ, V. G. E., SILVA, A. I. \& ARRUDA MIGUEL. Anthropometric profile and physical fitness of he professional referees chilean soccer. Int. J. Morphol., 26(4):897-904, 2008.

SUMMARY: The objective of the current study was to analyses the state of the anthropometric profile and physical ability of referees of first category participants of professional Chilean during the 2007 soccer Season. The sample was formed by 11 main referees, all male sex. The age was $34.54 \pm 4.76$ years old, height $1.74 \pm 0.05 \mathrm{~m}$, body mass $76.95 \pm 5.75 \mathrm{~kg}$ and $25.14 \pm 1.18 \mathrm{~kg} / \mathrm{m} 2 \mathrm{of}$ body mass index (BMI). The variables of body mass, height, skinfolds, body girth and bone diameters were collected with the aim of estimating the body composition and determining the somatotype of the referees. The new group of physical tests from FIFA determines to evaluate the referee first about his fitness to run quickly $(6 ¥ 40 \mathrm{~m})$ with a break of one minute and also to measure his ability of aerobic resistance in the second test. Regarding to the data of body composition the result indicate that the fat percentage is $15.44 \pm 2.81 \%$ with an average somatotype of $3.81-5.67-1.57$ classified in meso-endomorphic. The results show that the Chilean referees covered in average $5.32 \pm$ 0.02 the first test, meanwhile the second test all the referees covered easily distance of $4.000 \mathrm{~m}$. The higher body fat in the professional referees can be a limitation of the physical performance during the game that could be controlled by advisement and fitness programs.

KEY WORDS: Referee; Soccer; Anthropometry.

\section{REFERENCIAS BIBLIOGRÁFICAS}

Asami, T.; Togari, H. \& Ohashi, J. Analysis of movement patterns of referees during soccer matches. In: Reilly, T.; Lees, A.; Davids, K.; Murphy, W. J. eds. Sciense and Footboll. London, E. \& E N. Spon, 341-5, 1988.

Betsch, T. \& Plessner, H. Sequential effects in important referee decisions: the case of penalties in soccer. J. of sport \& Exercise Psychology, 23:254-9, 2001.

Callaway, C. W.; Chumlea, W. C.; Bouchard, C.; Himes, J. H.; Martin, A. D.; Mitchell, C. D.; Mueller, W. H.; Roche, A. F. \& Seefeldt, V. D. Circumferences. In. Lohman, T.G.; Roche, A.F. \& Martorell, R. Anthropometric standardization reference manual. Abridged edition. Human Kinetics Books. Champaign, Illinois. 1991.

Carter, J. E. L. The heath-carter anthropometric somatotype instruction manual. San Diego, USA. 2002.

Carter, J. E. L. \& Heath, B.H. Somatotyping-Development and Applications. Cambridge University Press, New York. 1990.

Carter, J. E. L.; Ackland, T. A.; Kerr, D. A. \& Stapff, A. B.
Somatotype and size of elite female basketball players. $J$. Sports Sci., 23(10):1057-63, 2005.

Casajus, J. A. \& Castagna, C. Aerobic fitness and field test performance in elite Spanish soccer referees of different ages. Journal of Science and Medicine in Sport, 133:1-8, 2006.

Castagna, C.; Abt, G. \& D’Ottavio, S. Competitive-level differences in yo-yo intermittent recovery and twelve minute run test performance in soccer referees. Journal of Strength and Conditioning Research. 19(4):805-9, 2005.

Castagna, C.; Abt, G. \& D’Ottavio, S. Relation Between Fitness Tests and Match Performance in Elite Italian Soccer Referees. J. Strength Cond. Res., 16 (2):231-5, 2002.

Castagna, C.; Abt, G.\& D'ottavio, S. Activity profile of international-level soccer referees during competitive matches. J. of Strength and Conditioning Research. 18 (3):486-90, 2004.

Catterall, C.; Reilly, T.; Atkinson, G. \& Coldwells, A. Analysis of the work rates and heart rates of association football referees. Br. J. Sp. Med., 27(3):193-6, 1993. 
Cuchiaro, A.L. Relação entre consumo/demanda energética, gordura corporal e estresse. Kinesis, (22):113$24,2000$.

Da Silva, A.I. Bases científicas e metodológicas para o treinamento do árbitro de futebol. Curitiba - Brasil, Imprensa da UFPR, 2005.

Da Silva, A. I. O IMC e o perímetro da cintura como indicadores de risco para a saúde de árbitros de futebol do Brasil. Fitness \& Perfornance Journal, 5(4):223- 31, 2006.

Da Silva, A. I. \& Fernández, R. Dehydration of football referees during a match. British Journal of Sport Medicine, Dez., 37:502-6, 2003.

Da Silva, A. I.; Fernandes, L. C. \& Fernández, R. Energy expenditure and intensity of physical activity in soccer referees during match-play. Journal of Sports Science and Medicine, 7: 327-34, 2008.

Da Silva, A. I.; Fernandes, L. C. \& Fernández, R. Índice de massa corporal e perímetros da cintura de árbitros de futebol da CBF. Rev. Educação Física/UEM. 18(1):41-47, 2007.

Da Silva, A. I. \& Nascimento, A. J. Composição corporal e aptidão física de árbitros da $\mathrm{CBF}$ submetidos à nova ordem de aplicação dos testes físicos da FIFA. Fitness \& performance J., 4 (5):306-12, 2005.

Da Silva, A. I. \& Rodriguez-Añez, C. R. Ações motoras do árbitro de futebol durante a partida. Treinamento Desportivo, Londrina: Editora Treinamento Desportivo, 4 (2):5-11, 1999.

Da Silva, A. I. \& Rodriguez-Añez, C.R. Cardiac responses to the physical activity of the soccer referee during a match. Cadernos Camilliani 8(1):83-90, 2007.

Da Silva, A. I. \& Rodriguez-Añez, C.R. Níveis de aptidão física e perfil antropométrico dos árbitros de elite do Paraná credenciados pela Confederação Brasileira de Futebol (CBF). Revista Portuguesa de Ciências do Desporto Portugual, 3(3):18-26, 2003.

Da Silva, A. I.; Romero, E. F.; Varanda, J. B. \& Santos, P. C. C. Capacidades físicas que deben ser desarrolladas durante um programa de entrenamiento para árbitros de fútbol. Santiago de Cuba. Revista Arrancada, 4 (5):53 - 61, 2002.
D’Ottavio, S. \& Castagna, C. Analysis of match activities in elite soccer referees during actual match play. $J$. of Srength and Conditioning Research. 15 (2):167$71,2001$.

Draper, N.; Whyte, G. Here's a new running-based test of anaerobic performance for which you need orly a stopwatch and a calculador. Peak Performance, 96: 3-5, 1997.

FIFA. Reglas de juego. Zurich. 2007.

Guerra, I.; Chaves, R.; Barros, T. \& Tirapegui, J. The influence of fluid ingestion on performance of soccer players during a match. Journal of Sports Science and Medicine, 3:198-202, 2004

Harrison, G. G.; Buskirk, E. R.; Carter, J. E. L.; Johnston, F. E.; Lohman, T. G.; Pollock, M. L.; Roche, A. F. \& Wilmore, J. H. Skinfold thicknesses and measurement technique. In. Lohman, T. G.; Roche. A. F.; Martorell, R. Anthropometric standardization reference manual. Abridged Edition. Human Kinetics Books. Champaign, Illinois, 1991.

Helsen, W. \& Bultynck, J.B. Physical and perceptualcognitive demands of top-class refereeing in association football. Journal of Sports Sciences. 22:179-89, 2004

Johnston, L. \& McNaughton, L. The physiological requirements of soccer refereeing. Aust. J. Sci. Med. Sport, 26(3-4): 67-72, 1994.

Krustrup, P. \& Bangsbo, J. Physiological demands of topclass soccer refereeing in relation to physical capacity: effect of intense intermittent exercise training. Journal of Sports Sciences, (19):881-91, 2001.

Krustrup, P.; Mohr, M.; Amstrup, T.; Rysgaard. T.; Johansen, J. \& Steensberg, A. The yo-yo intermittent recovery test: physiological response, reliability and validity. Med. Sci. Sports Exerc., 35(4):697-705, 2003.

Léger, L. A.; Mercier, D.; Gadoury, C. \& Lambert, J. The multiestage 20-meter shuttle run test for aerobic fitness. J. Sport Sci., 6:93-101, 1988.

Rahmawati, N. T.; Budiharjo, S. \& Ashizawa, K. Somatotypes of young male athletes and non-athlete students in Yogyakarta, Indonesia. Anthropological Science, 1-7, 2006. 
FERNÁNDEZ, V. G. E.; DA SILVA, A. I. \& ARRUDA MIGUEL. Perfil antropométrico y aptitud física de árbitros del fútbol profesional chileno. Int. J. Morphol., 26(4):897-904, 2008.

Rebelo, A.; Silva, S.; Pereira, N. \& Soares, J. Stress físico do árbitro de futebol no jogo. Revista Portuguesa de Ciências do Desporto. Portugual. Universidade do Porto, 2(5):24-30, 2002.

Rienzi, E.; Mazza. J. C.; Carter, J. E. L. \& Reilly, T. Futbolista Sudamericano de Elite: Morfología, Análisis del Juego y Performance. Rosario, Biosystem Servicio Educativo, 1998.

Roman, E. R.; Arruda, M.; Gasperin, C. E. B.; Fernandez, R. P. \& Da Silva, A. I. Estudo da desidratação, intensidade da atividade física e distância percorrida pelo árbitro de futebol durante a partida. Revista Brasileira de Fisiologia do Exercício, 3(2):160-71, 2004.

Rontoyannis, G. P.; Stalikas, A.; Sarros, G. \& Vlastaris, A. Medical, morphological and funcional aspects of Greek football referees. Journal of Sports Medicine and Physical Fitness, 38:208-14, 1998.

Weston, M.; Helsen, W.; MacMahon, C. \& Kirkendall, D. The impact of specific high-intensity training sessions on football referees' fitness levels. The American Journal of Sports Medicine, 32, 1 suppl.: 54s-61s, 2004.

Wilmore, J. H.; Frisancho, R. A.; Gordon, C. C.; Himes, J. H.; Martin, A. D.; Martorell, R. \& Seefeldt, V. D. Body Breadth Equipment and Measurement Techniques. In. Lohman, T.G.; Roche, A.F. \& Martorell, R. Anthropometric standardization reference manual. Abridged Edition. Human Kinetics Books. Champaign, Illinois, 1991.
Dirección para correspondencia:

Prof. Alberto Inácio da Silva

Almeda Nabuco de Araújo, 550, Apt. 10,

Bairro Uvaranas

CEP: 84031-510

Ponta Grossa, Paraná

BRASIL

Email: albertoinacio@bol.com.br

Recibido: 23-09-2008

Aceptado:25-10-2008 